\title{
Le sens méthodologique de la lecture de saint Paul dans l'Introduction à la phénoménologie de la religion de Heidegger
}

The Methodological Meaning of Saint Paul's Reading in Heidegger's

Introduction to the Phenomenology of Religion

El sentido metodológico de la lectura de San Pablo en la Introducción a la fenomenología de la religión de Heidegger

Laurent Villevieille

\section{OpenEdition}

\section{Journals}

Édition électronique

URL : http://journals.openedition.org/assr/27133

DOI : $10.4000 /$ assr.27133

ISSN : $1777-5825$

Éditeur

Éditions de l'EHESS

Édition imprimée

Date de publication : 1 septembre 2015

Pagination : 213-240

ISBN : 9-782713224706

ISSN : 0335-5985

\section{Référence électronique}

Laurent Villevieille, « Le sens méthodologique de la lecture de saint Paul dans l'Introduction à la phénoménologie de la religion de Heidegger », Archives de sciences sociales des religions [En ligne], 171 | 2015, mis en ligne le 01 septembre 2018, consulté le 19 avril 2019. URL : http:// journals.openedition.org/assr/27133; DOI : 10.4000/assr.27133 


\section{Le sens méthodologique de la lecture de saint Paul dans l'Introduction à la phénoménologie de la religion de Heidegger}

Le cours que Heidegger prononce au semestre d'hiver 1920-1921, et qui figure en tête du tome 60 de l'Édition intégrale, est intitulé : Introduction à la phénoménologie de la religion ${ }^{1}$. Après une introduction méthodologique, une bonne moitié du cours s'attache à expliquer trois épîtres de saint Paul : l'épître aux Galates, et les deux épîtres aux Thessaloniciens. D'emblée, une question très simple se pose au lecteur : en quoi l'explication de ces épîtres pauliniennes constitue-t-elle une "introduction à la phénoménologie de la religion » ? Cela revient d'abord à demander, d'une manière générale, pourquoi Heidegger introduit ses étudiants à la phénoménologie de la religion par l'explication d'un texte sacré, plutôt que, par exemple, par une analyse du concept de religion ou de l'histoire du christianisme. Cette question, nous ne la traiterons qu'indirectement, et pour ainsi dire de biais ${ }^{2}$. Car le cours de Heidegger pose une autre question qui, dans une certaine mesure, sera à même d'éclairer rétroactivement la première : pourquoi saint Paul ? En effet, pourquoi saint Paul plutôt que saint Jean, Moïse ou Salomon ? En somme, qu'est-ce que la parole paulinienne peut avoir d'exemplaire pour la phénoménologie de la religion - de telle sorte que cette exemplarité assure en même temps le caractère introductif du cours ?

Assurément, une nouvelle question pourrait être posée, plus précise encore, mais aussi, en un sens, plus élémentaire : pourquoi ces épîtres-là ? Qu'est-ce que Heidegger trouve dans l'épître aux Galates et dans les deux épîtres aux Thessaloniciens, qu'il ne trouve pas, ou pas aussi magistralement, dans les épîtres aux Romains ou aux Philippiens ? Répondre à cette dernière question nous entraînerait sur un tout autre terrain que ne le fait la question que nous posons - celle

1. Einleitung in die Phänomenologie der Religion, in Phänomenologie des religiosen Lebens, Gesamtausgabe, Band 60, Frankfurt am Main, Vittorio Klostermann, 1995 (désormais noté GA 60), p. 1-156. Trad. fr. Introduction à la phénoménologie de la religion, in Phénoménologie de la vie religieuse, Paris, Gallimard, 2011, p. 11-174.

2. Pour un traitement approfondi de cette question, on se reportera à l'ouvrage classique de P. Capelle, Philosophie et théologie dans la pensée de Martin Heidegger, Paris, Cerf, 2001. 
de l'exemplarité de la parole paulinienne. Nous serions alors conduits à étudier notamment la «kairologie » paulinienne, c'est-à-dire l'expérience non chronologique du temps que décrit en particulier la première épître aux Thessaloniciens, et qui nourrit forcément la réflexion d'un penseur qui sera amené à écrire un ouvrage intitulé Être et Temps. Ces questions ont déjà été traitées - depuis les études, à l'époque inédites, qu'Otto Pöggeler avait consacrées au manuscrit du cours de 1920-1921 auquel il avait eu accès ${ }^{3}$, jusqu'à l'étude plus récente de Sophie-Jan Arrien ${ }^{4}$. Notre question directrice doit nous conduire à des questions moins thématiques et, pour tout dire, plus méthodologiques - celle de savoir ce que la lecture de Paul peut apporter, précisément, à la méthode phénoménologique en général, et plus particulièrement à la fondation d'une phénoménologie de la religion.

Puisque le cours de 1920-1921 vise à y introduire, il semblerait opportun, si l'on veut mesurer si et jusqu'à quel point l'explication de la parole paulinienne le permet, de définir au préalable ce qu'est la phénoménologie, et, plus particulièrement encore, ce qu'est la phénoménologie de la religion. En réalité, nous verrons que c'est la parole paulinienne elle-même, du moins telle que Heidegger l'explique et la comprend, qui permet inversement de dessiner dans ses grandes lignes le sens et la portée d'une phénoménologie de la religion et, d'un seul et même tenant, du discours phénoménologique comme tel, dont cette parole s'avère singulièrement exemplaire.

\section{1. "La tentation de prélever certains concepts "}

Au $\ 16$, que l'éditeur du cours a intitulé : «L'attitude fondamentale de Paul », Heidegger met en garde son auditeur contre une tendance de lecture très répandue :

Quand on étudie le monde religieux de Paul, on doit se libérer de la tentation de prélever certains concepts (tels que $\pi i \sigma \tau \iota \zeta, \delta \iota x \alpha \iota \sigma \sigma u ́ v \eta, \sigma \alpha ́ \varrho \xi$, <foi, justice, chair>, etc.) et de déduire leur signification en combinant des passages isolés des écrits pauliniens, de sorte qu'on obtient un catalogue de concepts fondamentaux qui ne veulent rien dire. Tout aussi erronée est l'idée selon laquelle il y aurait un système théologique de Paul. Il faut au contraire mettre en évidence l'expérience religieuse fondamentale de Paul et, en se maintenant dans cette expérience fondamentale, tenter de comprendre la connexion de tous les phénomènes religieux originels avec celle-ci (GA 60, p. 73 ; trad. fr. p. 83).

La lecture heideggérienne de Paul se définit d'abord négativement : «se libérer de la tentation de prélever certains concepts ». Tout le problème est ici de

3. O. Pöggeler, Der Denkweg Martin Heideggers, Pfüllingen, G. Neske, 1963 (trad. fr. M. Simon, La pensée de Martin Heidegger. Un cheminement vers l'être, Paris, Aubier-Montaigne, 1967, p. 47-59).

4. S.-J. Arrien, «Foi et indication formelle. Heidegger, lecteur de saint Paul », in S.-J. Arrien et S. Camilleri (dir.), Le jeune Heidegger (1909-1926), Paris, Vrin, 2011, p. 155-172. 
savoir ce que signifie : "prélever " (herausgreifen). Car enfin, pour lire un texte, on est bien obligé de s'arrêter sur une expression plutôt que sur une autre - par exemple sur le mot « chair » plutôt que sur les mots « il » ou « à ». En général, ce choix est censé être justifié par le poids philosophiquement particulier de certaines expressions : on parle alors de "concepts ». Heidegger met donc en garde son auditeur contre la «tentation» de se laisser en quelque sorte capter par des expressions philosophiquement attirantes. "Prélever " signifierait donc: détacher de son contexte, isoler et peut-être, par suite, monter en épingle (sous forme d'un " catalogue de concepts fondamentaux qui ne veulent rien dire »). La question qui, dès lors, se pose tout naturellement est : qu'est-ce que ne pas " prélever certains concepts" ? N'est-on pas toujours contraint, lorsqu'on explique un texte (comme c'est aussi notre propre cas en ce moment), d'opérer une sélection de certaines expressions au détriment d'autres ? Heidegger enjoint au contraire de «mettre en évidence l'expérience religieuse fondamentale de Paul ». Il y aurait donc quelque chose, non pas derrière le texte, mais plutôt au cour du texte, qui devrait être dévoilé par le texte lui-même, mais que l'on risquerait à tout moment de rater si l'on réduit le texte à un ensemble de pièces détachées, celles-ci fussentelles nommées "concepts fondamentaux ». À ces " concepts » prétendument "fondamentaux ", Heidegger oppose des "phénomènes religieux originels ". Cependant, la question rebondit : quelle est la différence entre des « concepts » et des "phénomènes ", du moment que ces derniers sont saisis philosophiquement ? Qu'est-ce qui permet à l'approche phénoménologique des "phénomènes » de ne pas dégénérer en "catalogue de concepts " ? Ce que Heidegger nomme ici : " connexion " (Zusammenhang). C'est en étant saisis tous ensemble que les phénomènes échappent au danger d'être des concepts simplement "prélevés ».

Mais avant de demander comment des phénomènes pourraient bien être saisis tous ensemble ${ }^{5}$, une question plus pressante se pose à nous. Notre objectif était en effet de déterminer ce que la parole paulinienne pouvait avoir d'exemplaire pour une introduction à la phénoménologie de la religion. Or la mise en garde de Heidegger au sujet de la «tentation de prélever certains concepts » pourrait, semble-t-il, s'appliquer à la lecture de n'importe quel texte. En quoi les remarques précédentes, qui semblent s'apparenter à une méthodologie générale de l'explication de texte philosophique, peuvent-elles bien nous éclairer sur la spécificité de la parole paulinienne? On dira que Heidegger parle de phénomènes religieux, ou qu'il met en garde son auditeur contre le danger de voir dans la parole paulinienne une théologie. Soit - mais ne pourrait-il pas développer exactement le même propos au sujet de n'importe quel texte sacré ?

5. C'est à ce problème de la totalité que se confrontera également Être et Temps, traité dans lequel Heidegger tentera de saisir la structure unitaire de l'être-au-monde sur un mode analytique, c'est-à-dire sur un mode qui, sous le titre d' " analytique existentiale », en dissocie les trois moments structuraux ( "monde ", «soi » et «être-à ») tout en en préservant l'unicité structurale. Sur ce point, qu'il nous soit permis de renvoyer à notre ouvrage Heidegger et l'indétermination d'Être et Temps, Paris, Hermann (coll. « Le Bel Aujourd'hui »), 2014. 


\section{Doctrine ancienne et expérience nouvelle}

Le texte que nous avons cité suit de près un autre passage capital du cours, qui figure dans le même $\mathbb{} 16$ :

Paul est engagé dans un combat. Il est obligé d'affirmer l'expérience chrétienne de la vie contre le monde ambiant. Pour cela, il emploie les moyens inadéquats de la doctrine rabbinique qu'il a à sa disposition. C'est cela qui donne sa structure particulière à son explication de l'expérience chrétienne de la vie. Néanmoins, il s'agit d'une explication originelle, tirée du sens de la vie religieuse elle-même. Elle peut recevoir une élaboration plus poussée dans l'expérience religieuse fondamentale. Les connexions théoriques sont maintenues à l'écart; mais on y gagne une connexion d'explicitation qui ressemble à une explicitation théorique (GA 60, p. 72 ; trad. fr. p. 82, trad. modifiée).

Nous avons vu précédemment qu'il importait, dans la lecture d'un texte sacré (et peut-être plus généralement de n'importe quel texte), de reconduire le texte à l'expérience originaire dont il provient - donc ici, comme le précisait le texte précédemment cité, "l'expérience religieuse fondamentale de Paul ». Une telle reconduction passe, bien entendu, par le texte lui-même. Or la parole paulinienne a ceci de particulier, qu'elle emploie des moyens "inadéquats " : ceux de la "doctrine rabbinique ». On sait en effet - et le texte même de l'épître aux Galates que Heidegger est alors en train d'expliquer le rappelle - qu'avant de se convertir,

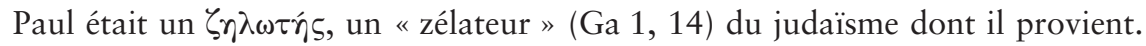
Or s'il s'est converti, le discours même de conversion garde la trace de cette provenance. C'est ainsi dans un langage ancien que Paul annonce l'expérience nouvelle, c'est-à-dire, plus précisément, la «bonne nouvelle » qu'est l'Évangile $\mathrm{du}$ Christ. La parole paulinienne est pour ainsi dire en retard sur ce qu'elle nomme. En ce sens, elle est « inadéquate ». Mais en quoi cette inadéquation, loin d'être ici un défaut, est-elle exemplairement introductrice à la phénoménologie de la religion ? On a vu que la phénoménologie doit «se libérer de la tentation de prélever certains concepts ». Or cette tentation n'est évidemment jamais aussi grande que là où le texte lui-même semble y inciter. Tel est le cas du texte paulinien qui, parce qu'il a recours à la "doctrine rabbinique ", c'est-à-dire à une Lehre, à une "doctrine ", donc à quelque chose qui a toute l'apparence d'un contenu " théorique ", semble inciter à l'attitude, elle-même théorique, de repérage des concepts fondamentaux. Et cependant, la parole paulinienne n'est théorique qu'en apparence : elle ne fait, précise Heidegger, que "ressembler à une explicitation théorique ». En réalité, "les connexions théoriques sont maintenues à l'écart ». Pourquoi ? Parce que sous l'apparence d'un vocabulaire doctrinal, la parole paulinienne est « une explicitation originelle, tirée du sens de la vie religieuse ellemême ». Qu'en conclure?

Que la parole paulinienne place le phénoménologue dans la situation typique d'une phénoménologie de la religion (et peut-être, comme nous le verrons, de la phénoménologie en général). C'est-à-dire dans la situation de devoir aller chercher le sens du phénomène religieux dans ce qui, constamment, menace de se constituer en doctrine théorique, de se solidifier et de se réifier en un ensemble 
de concepts vides. Car la parole paulinienne a cette ambivalence : d'apparence théorique, elle dit tout autre chose que du théorique.

\section{Contenu dogmatique et proclamation}

Le danger que comporte le texte paulinien, Heidegger le réfère encore à une autre dimension de ce texte - ou plus exactement à la même dimension, mais appréhendée sous un autre angle. Dans un passage capital, qui va du reste nous permettre de jeter une certaine lumière sur la relation que la phénoménologie de la religion peut entretenir avec l'exégèse, et par suite avec la théologie, Heidegger note en effet :

L'épître aux Galates a un contenu «dogmatique ». En exégèse, c'est celui-ci qu'on voit tout d'abord. Mais on doit être au clair avec la manière dont il faut comprendre ce contenu comme un "savoir croyant ». Ce que dit Paul se caractérise par le fait que c'est maintenant qu'il le dit aux Thessaloniciens, ou encore aux Galates. Il ne faut pas se précipiter vers le contenu isolé. Il faut comprendre le prétendu contenu dogmatique des épîtres dans l'ensemble de la manière dont on obtient une communication du savoir chrétien. En l'abordant à l'état isolé, on s'égare (GA 60, p. 101-102 ; trad. fr. p. 113-114).

Heidegger commence par affirmer que "l'épître aux Galates a un contenu “dogmatique" ». Et en effet, on sait l'importance que saint Paul a eue dans la constitution de la dogmatique chrétienne. Insister sur cette propension de la parole paulinienne à se constituer en dogmatique, c'est remarquer à nouveau, mais sur un autre mode, l'apparence théorique que Heidegger avait déjà repérée dans la parole paulinienne. Ce n'est cependant, nous l'avons dit, qu'une apparence. C'est pourquoi Heidegger place ici le mot «dogmatique » entre guillemets - pour parler finalement, quelques lignes plus bas, d'un " prétendu contenu dogmatique ». Si ce « contenu dogmatique » n'est que "prétendu », c'est que, pour être saisi de la sorte, il faut qu'il ait été abordé "à l'état isolé » (isoliert). "Isoler » fait ici écho à l'expression du texte précédent : "prélever ». Ainsi, dans l'épître aux Galates, on obtiendra un contenu dogmatique par exemple en isolant les deux concepts de foi et de loi, et en établissant sur un mode formel la teneur de leur relation.

À cette attitude abstractive, Heidegger oppose un retour à un mystérieux "maintenant»- celui de la proclamation paulinienne. Heidegger prônerait-il une lecture contextuelle de Paul ? À vrai dire, l'enracinement de la proclamation paulinienne dans son propre «maintenant» n'est pas ici le fait du lecteur : il est bien plutôt le fait de la parole paulinienne elle-même. Cet enracinement est en effet, note Heidegger, ce qui " caractérise » la parole paulinienne elle-même ( «Ce que dit Paul se caractérise par le fait que c'est maintenant qu'il le dit aux Thessaloniciens, ou encore aux Galates »). Mais comment une parole pourraitelle «se caractériser " par son propre "maintenant» ? En étant une parole dont le caractère doctrinal ou théorique n'est qu'un moyen inadéquat pour saisir tout 
autre chose qu'une doctrine ou une théorie : à savoir le «maintenant » de la proclamation. C'est de la richesse de ce «maintenant » que le «prétendu contenu dogmatique » est abstrait. Heidegger, sans limiter l'exégèse à cette attitude abstractive, note toutefois qu' "en exégèse, c'est celui-ci [i.e. le prétendu contenu dogmatique] qu'on voit tout d'abord ». Si donc l'exégèse n'a pas intrinsèquement et par définition vocation à se cantonner au "prétendu contenu dogmatique ", elle est toutefois grandement exposée à ce risque, puisque c'est un tel contenu qui s'impose «tout d'abord » à elle.

\section{Abstraction et explicitation}

Deux attitudes semblent alors se dessiner, dont la parole paulinienne, par son ambivalence, pousse à préciser les contours : d'une part, l'attitude abstractive qui, sans être celle de l'exégèse, guette constamment celle-ci; d'autre part, une attitude que Heidegger qualifie d'un terme qui, plus tard, viendra souvent se substituer au mot Auslegung, " explicitation ", qui caractérise la méthode herméneutique : il s'agit du mot Explikation, qui signifie donc moins «explication » que, comme le note judicieusement J. Greish dans sa traduction, " explicitation ». Voici le passage qui exprime sans doute le plus clairement l'opposition de l'abstraction et de l'explicitation :

Ce qui est abstrait est soumis à une considération ultérieure, sans que soit pris en considération ce dont il est abstrait ; le fundamentum de l'abstraction nous est indifférent. L'abstraction en tant que telle, le passage du fundamentum abstractionis à l'acte d'abstraire n'est pas expérimenté en même temps. Il en va autrement de l'explicitation (Explikation) : si, au cours de l'explicitation, certains moments déterminés sont explicités (expliziert), les moments de sens sur lesquels l'explicitation ne s'oriente pas ne sont pas simplement mis de côté ; au contraire, le "comment " de leur pénétration dans la direction de sens explicitée à ce moment précis, ou bien dans l'explicitation, voilà ce qui est justement codéterminé par l'explicitation elle-même (GA 60, p. 86 ; trad. fr. p. 97).

Il faut renoncer ici à suivre le fil de la référence, à peine voilée, à la doctrine husserlienne de l'abstraction, développée notamment dans la deuxième Recherche logique. Concentrons-nous plutôt sur les conclusions qu'elle permet à Heidegger de tirer. Lorsque j'abstrais un concept général d'un individu (par exemple le concept de rouge de ce canapé rouge), le concept que j'abstrais (ici : le rouge) est parfaitement « indifférent » au "fundamentum de l'abstraction » (c'est-à-dire, dans notre exemple, au canapé rouge). Peu importe que je me fonde sur un canapé, une pomme ou une tulipe pour en abstraire le concept général de rouge. Car en tant que concept général, le concept de rouge n'appartient à aucun individu en particulier.

Tel est le procédé de lecture contre lequel Heidegger met en garde ses auditeurs depuis le début : abstraire du texte paulinien les concepts de foi, de justice ou de chair comme on abstrait le concept de rouge d'un canapé, d'une pomme 
ou d'une tulipe. Donc comme si ces concepts étaient détachables de leur « fundamentum ». Mais ce "fundamentum ", justement, quel est-il ? Le texte de Paul ? Plutôt l'expérience religieuse fondamentale dont la parole paulinienne provient. Cette parole doit être reconduite à cette expérience. Non pas, là encore, parce qu'une bonne méthode de lecture l'exige. Mais parce que la parole paulinienne elle-même le nécessite, en tant que parole qui n'a de doctrinal et de théorique que l'apparence - puisque doctrine et théorie ne sont pour elle qu'un moyen de dire, en termes anciens, donc inadéquats, une expérience nouvelle.

À la tentation de l'abstraction, Heidegger oppose la méthode de l'explicitation. Opérer une abstraction, c'est laisser de côté les «moments de sens » avec lesquels coexiste le concept que l'on abstrait. Par exemple, pour abstraire le concept de rouge d'un canapé rouge, je vais laisser de côté la forme du canapé, sa fonction, etc. - toutes choses qui n'ont strictement rien à voir avec le concept général de rouge. En revanche, lorsque j'explicite, «les moments sur lesquels l'explicitation ne s'oriente pas ne sont pas simplement mis de côté ". Ainsi, ce ne sera pas parce que j'explique le mot " foi » que le « caractère épistolaire » (GA 60, p. 81 ; trad. fr. p. 91) des écrits de Paul sera perdu de vue. Au contraire, le moment de sens «foi » sera compris à la lumière de ce caractère épistolaire (donc à la lumière de sa proclamation à une communauté de croyants, etc.). C'est au fond ce à quoi Heidegger veut inviter ses auditeurs en les enjoignant d'" envisag[er] la situation comme si nous écrivions l'épître ensemble avec Paul » (GA 60, p. 87 ; trad. fr. p. 99).

\section{Lecture et indication formelle}

Le mode de lecture qu'appelle la parole paulinienne trouverait ainsi sa traduction phénoménologique dans un concept de méthode que développe la première partie du cours de 1920-1921: le concept d'indication formelle ${ }^{6}$. À l'époque où le cours fut prononcé, les auditeurs de Heidegger étaient allés se plaindre au doyen de l'université de ce que, s'étant inscrits à un cours de philosophie de la religion, ils n'y entendaient parler que de méthode phénoménologique ${ }^{7}$. C'est en réponse à cette plainte que Heidegger décida, non sans irritation, de se concentrer sur l'étude des épîtres pauliniennes, étude qu'il introduisit dans les termes suivants :

6. Sur ce concept méthodologique fondamental dans la pensée du jeune Heidegger, on pourra se reporter à S.-J. Arien, art. cit., qui s'attache particulièrement à montrer la relation entre l'indication formelle et la lecture heideggérienne de saint Paul, ainsi qu'à notre étude " Heidegger, de l'indication formelle à l'existence » (Bulletin d'Analyse Phénoménologique, IX, 5 , p. 1-96), qui insiste davantage sur la relation Husserl-Heidegger.

7. Sur ce point, on pourra consulter la postface de l'éditeur allemand (GA 60, p. 339-340; trad. fr., p. 385), ainsi que le récit que fait T. Kisiel de cet épisode dans son article « L'indication formelle de la facticité : sa genèse et sa transformation ", in J.-F. Courtine (dir.), Heidegger 1919-1929. De l'herméneutique de la facticité à la métaphysique du Dasein, Paris, Vrin, 1996, p. 210-211. 
La philosophie, telle que je la conçois, se trouve prise dans une difficulté. L'auditeur d'autres cours est rassuré d'emblée : dans un cours d'histoire de l'art, il peut contempler des images, dans d'autres il rentre dans ses frais en passant un examen. En philosophie, il en va autrement, et je n'y peux rien, car ce n'est pas moi qui ai inventé la philosophie. Je voudrais pourtant me sauver de cette calamité, et c'est pourquoi j'interromprai ces considérations si abstraites pour vous faire, à partir de la prochaine heure, un cours d'histoire. Je partirai d'un phénomène concret déterminé, sans m'attarder davantage à des considérations d'approche et de méthode, mais, en ce qui me concerne, en présupposant que, du début jusqu'à la fin, vous comprendrez de travers l'ensemble de mes réflexions (GA 60, p. 65 ; trad.fr. p. 75, trad. modifiée).

Au-delà de l'opposition des «considérations abstraites » et du "phénomène concret déterminé », de la "philosophie » et de l' « histoire », les épîtres pauliniennes ont la vertu phénoménologiquement insigne d'inviter leur lecteur à saisir la parole sur le mode d'une indication formelle. Disons en effet très sommairement que l'indication formelle consiste à n'avoir recours à des concepts, ou formes (dans le cas de Paul : la "doctrine rabbinique ») que pour indiquer quelque chose qui n'est pas de l'ordre conceptuel (pour Paul, le «maintenant » de la proclamation) et que l'explicitation ne peut dès lors atteindre qu'en dénonçant son propre caractère conceptuel (ce dont se charge Heidegger lecteur de Paul). Dans les termes que Heidegger a adoptés dans sa lecture des épîtres, un concept isolé, dès le moment où il indique la totalité de sens dont il provient, cesse d'être un concept isolé. Ces termes vaudraient au fond pour tout concept phénoménologique.

Au terme de notre étude, la signification d'une phénoménologie de la religion commence à se dessiner. On a vu un peu plus haut que l'exégèse, si elle ne se définissait évidemment pas par la tentation d' "isoler » des concepts, était " avant tout » confrontée à cette tentation. Pourquoi ? Parce que c'est d'un tel isolement que peut procéder l'énoncé d'un contenu dogmatique. La phénoménologie de la religion se définit ainsi comme un retour à la totalité de sens dont proviennent les concepts décharnés de la dogmatique. Elle est, si l'on veut, l'herméneutique que toute exégèse doit garder auprès de soi pour ne pas dégénérer en l'énoncé d'un simple « catalogue de concepts ». Dès lors, la théologie se tient dans l'alternative que constituent la phénoménologie de la religion et la dogmatique :

Le savoir relatif au propre être-devenu est le point de départ et l'origine de la théologie (GA 60, p. 96 ; trad. fr. p. 107).

«Être-devenu » est ici l'expression formelle de ce que Heidegger avait par ailleurs nommé " expérience religieuse fondamentale ». On dirait plus couramment : la conversion. La théologie doit assumer son enracinement dans cette expérience fondamentale. Et la phénoménologie de la religion a à charge de la rappeler à cet enracinement, c'est-à-dire à la totalité de sens dont proviennent les concepts théologiques. 
La parole paulinienne a alors ceci d'exemplaire, pour la phénoménologie de la religion, qu'elle se situe pour ainsi dire dans l'entre-deux : exprimée à partir de la "doctrine rabbinique ", elle dit tout autre chose qu'une doctrine. Elle est ainsi paradigmatique de ce que la phénoménologie de la religion se doit d'accomplir : une reconduction du théorique à l' «être-devenu » qui, lui, n'est rien de théorique.

Laurent VILLEVIELLE

Archives Husserl de Paris

laurentvillevieille@gmail.com

\section{Le sens méthodologique de la lecture de saint Paul dans l'Introduction à la phénoménologie de la religion de Heidegger}

Pour introduire ses étudiants à la phénoménologie de la religion, le jeune Heidegger décide d'expliquer trois épîtres de saint Paul. En quoi l'explication de la parole paulinienne peut-elle assumer cette fonction introductrice? Aux yeux de Heidegger, la parole de saint Paul est une parole de l'entre-deux : empruntant son vocabulaire à la doctrine ancienne, elle tente de dire l'expérience nouvelle. Comment atteindre l'expérience au travers d'une doctrine et, par suite, le nouveau au travers de l'ancien? Comment la parole peut-elle rattraper son retard sur ce qu'elle nomme? Telles sont les questions phénoménologiquement exemplaires qui se posent à celui qui tente d'expliquer la parole paulinienne, et qui, en y répondant, s'initie à la phénoménologie de la religion.

Mots-clés : Heidegger, saint Paul, méthode phénoménologique, exégèse, dogmatique, explicitation, indication formelle.

\section{The Methodological Meaning of Saint Paul's Reading in Heidegger's Introduction to the Phenomenology of Religion}

In order to introduce his students to the phenomenology of Religion, young Heidegger decides to expound on three epistles of Saint Paul. How can the analysis of the Paulinian discourse assume this introductory function? In the eyes of Heidegger, Saint Paul's discourse is an in-between discourse: borrowing its vocabulary to an old doctrine, it tries to speak of a new experience. How to attain experience through doctrine, and consequently, the new through the old? How can discourse amend its own delay towards what it names? These are the exemplary phenomenological questions that are being asked to anyone trying to explain the Paulinian discourse, and who, by answering these questions, is being initiated to the Phenomenology of Religion.

Key words: Heidegger, Saint Paul, phenomenological method, exegesis, dogma, elicitation, formal indication. 


\section{El sentido metodológico de la lectura de San Pablo en la Introducción a la fenomenología de la religión de Heidegger}

Para introducir a sus estudiantes a la fenomenología de la religión, el joven Heidegger decide explicar tres epístolas de San Pablo. ¿En qué sentido la explicación de la palabra paulina puede asumir esta función introductoria? A los ojos de Heidegger, la palabra de San Pablo es una palabra del entre-dos: tomando su vocabulario de la doctrina antigua, trata de decir la experiencia nueva. ¿Cómo llegar a la experiencia a través de una doctrina y, luego, lo nuevo a través de lo viejo? ¿De qué maneras la palabra puede recuperar su atraso sobre aquello que nombra? Estas son las preguntas fenomenológicamente ejemplares que se plantean a quien trata de explicar la palabra paulina y que, en la búsqueda de respuestas, se inicia en la fenomenología de la religión.

Palabras clave: Heidegger, San Pablo, método fenomenológico, exégesis, dogmática, explicitación, indicación formal. 